\title{
Optimizing Fuzzy Rule Base for Illumination Compensation in Face Recognition using Genetic Algorithms
}

\author{
Bima Sena Bayu Dewantara(1,2), Jun Miura(2) \\ (1) Department of Informatics and Computer Science, Electronic Engineering \\ Polytechnic Institute of Surabaya, Indonesia \\ Jl. Raya ITS, Keputih, Sukolilo, Surabaya, 60111, (031)5947280 \\ (2) Department of Computer Science and Engineering, Toyohashi University of \\ Technology, Japan \\ 1-1 Hibarigaoka, Tenpaku-Cho, Toyohashi-Shi, 441-8580 \\ E-mail: bima@pens.ac.id
}

\begin{abstract}
Fuzzy rule optimization is a challenging step in the development of a fuzzy model. A simple two inputs fuzzy model may have thousands of combination of fuzzy rules when it deals with large number of input variations. Intuitively and trial-error determination of fuzzy rule is very difficult. This paper addresses the problem of optimizing Fuzzy rule using Genetic Algorithm to compensate illumination effect in face recognition. Since uneven illumination contributes negative effects to the performance of face recognition, those effects must be compensated. We have developed a novel algorithmbased on a reflectance model to compensate the effect of illumination for human face recognition. We build a pair of model from a single image and reason those modelsusing Fuzzy.Fuzzy rule, then, is optimized using Genetic Algorithm. This approachspendsless computation cost by still keepinga high performance. Based on the experimental result, we can show that our algorithm is feasiblefor recognizing desired person under variable lighting conditions with faster computation time.
\end{abstract}

Keywords: Face recognition, harsh illumination, reflectance model, fuzzy, genetic algorithm

\section{INTRODUCTION}

Fuzzy logic, initially introduced by Zadeh [1] in mid-1960s has been widely used in control engineering. More recently, it has widely explored and developed to solve many problems in a wide scope of science as well as engineering field. Fuzzy logic is a mathematical theory of inexact reasoning that allows us to model the reasoning process of humans in linguistic terms. It is suitable in defining the relationship between the system inputs and the desired system outputs. 
The main strength of fuzzy logic is its rule base. The fuzzy rule base is defined as a conditional statement of a linguistic variable. The rule base is used to map the input to the desired output. It is usually determined intuitively and trial-error. However, designing a fuzzy rule base with large amount of input variations is not a trivial problem. To deals with this problem, an optimization technique must be used.

Optimization is the most common problem to find a set of solution that is optimal from many possible solutions. Finding an optimal solution is related to the subject to be optimized. Each subject needs different treatment and approach. Genetic Algorithm (GA) [2] is the most popular optimization algorithm in evolutionary algorithms.GA is based on genetic science and natural selection to simulate the phenomenon of natural evolution at genotype level. It is widely used and successfully solving problems in many fields.

By adopting the GA's way in our fuzzy rule base, we optimize our illumination compensation model [3] for robust face recognition in a harsh illumination environment.

\subsection{RELATED WORKS}

Genetic algorithm has been showed its superiority in fuzzy optimization problems. Applyinggenetic algorithm for optimizing fuzzy rule base is better than manually determine the fuzzy rule for large number of input and output patterns. Genetic algorithm has been widely and successfully used to optimize fuzzy rules in control problems between 1995-2005. Since fuzzy, then, is adopted in image processing and computer vision problems, the genetic algorithm is also used in this area of interests.

Several methods based on fuzzy approach have been proposed to deal with illumination compensation problems for face recognition. Most of them are based on photometric normalization technique; atechnique uses human perception theory and illumination properties. Photometric normalization based approaches, i.e., Retinex-theory and Quotient-Image become more acceptable. Retinex-theory deals with illumination effect on images. It estimates the image's reflectance to achieve a color constancy/lightness rendition. While, the Quotient-Image is an image ratio between a test image and a linear combination of three images illuminated by non-coplanar lights and depends only on the albedo information.

Nam [4]introduced the use of Fuzzycombined with Retinex method to compensate the illumination on a mobile device. Their method determinesthe optimal parameters of Retinex filteringusing Fuzzy.The output of Fuzzy is adaptively determined by themean and standard deviations of the gray values.Unfortunately, the same as the standard retinex, the result frequently become very blurred. A major improvement is achieved by Adaptive Smoothing Retinex (ASR) [5] where the illumination can be estimated by iteratively convolving the input image with a $3 \times 3$ smoothing mask weighted by coefficient via combining two measures of the illumination 
discontinuity at each pixel. It produces a better converted image but spends a lot of computation time.

Perez and Castillo [6] introduced an Optimized-SQI (Opt-SQI) to optimize SQI's parameters using Genetic Algorithm (GA). GA optimizes the mean value within the region for the SQI, selection of optimal non-linear function and the values for the weights of each filter. Since the Opt-SQI combines many Gaussian filters, it requires a very high cost and still experiences strong edge artifacts at boundaries.

More recently, a Fuzzy-based approach was proposed by Singh [7]. They first classified appearance variations into dark, normal or shadow using four statistical parameters (average mean level, standard deviation, uniformity and entropy) which are used as input of rough membership function ( $\mathrm{rmf}$ ) classifier, and selects an illumination compensation (HE, Homomorphic Filter or SQI) based on the decided illumination type. It works very fast but it may produce different appearance of each converted image because each illumination compensation method has different final appearances. Therefore, it needs more training data per subject for each method.

\subsection{OUR APPROACH}

We have introduced a novel technique to get an appropriate illumination ratio $r$ by using a pair of model fromsingle image only [3]. The first model is used to approximate the nativeface appearance by roughly equalizingthe image's contrast. The second model is used to present actual face appearance from the image. By using Fuzzy InferenceSystem (FIS) [8], we can reason those models to obtain an illumination ratio $r$ whichis optimum for our illumination compensation model. We utilize Genetic Algorithm inorder to optimize FIS rules to produce appropriate $r$ for each condition. Byapplying optimized rinto our illumination compensation model, a harsh illuminated face image can be converted into ashadow-free and specular reflection-free face image.

In this paper, we are focusing our work on optimizing Fuzzy rules using Genetic Algorithm instead of our illumination compensation method and its experimental works associated with this optimization.

\subsection{PAPER ORGANIZATION}

The rest of this paper is organized as follows: We briefly describe our illumination compensation framework in section II, followed by our Fuzzy optimization in section III. Then, experiments and discussion is presented in section IV. And finally, conclusion of our work and possible future work are described in section $V$.

\section{ILLUMINATION COMPENSATION FRAMEWORK}

In this section, we will briefly explain the illumination compensation model by [9] and our modification to fit our problem. 


\subsection{OUR ILLUMINATION COMPENSATION MODEL}

The illumination compensation model by [9] is expressed as:

$$
I(x, y)=\frac{(r+1)}{(k(x, y) r+1)} I^{*}(x, y)
$$

where $I(x, y)$ is the normalized intensity of pixel $(\mathrm{x}, \mathrm{y}), r$ is the illumination ratio, $k(x, y)$ is the label of pixel $(\mathrm{x}, \mathrm{y})$ and $I^{*}(x, y)$ is the raw intensity of pixel $(\mathrm{x}, \mathrm{y})$. They determined the illumination ratio $r$ using the intensities of two neighboring pixels of different segments. These different segments are caused by the presence of direct illumination $L_{d}$ and environment illumination $L_{e}$. The illumination ratio can simply be obtained from $r=$ $L_{d} / L_{e}$. The pixel's label $k(x, y)$ is an inherent characteristic to the direct illumination; angle of incidence $\theta(x, y)$ and other factors $d(x, y)$ which affect the amount of light to the surface. Then, the pixel's label can be expressed as $k(x, y)=d(x, y) \cos (\theta(x, y))$. Since they focused on shadow and non-shadow areas, $0 \leq k(x, y) \leq 1$ is used.

This approach is certainly failing when it could not find a pair of different segments (caused by $L_{d}$ and $L_{e}$ ) to be classified. We have investigated that the problem of illumination on the human face is quite unique and very far different from those with landscapes. In several extreme illumination conditions, e.g., backside illumination, one-side illumination, or very low illumination, getting pairs of pixels from adjacent boundary is difficult. Therefore, we propose a new approach to determine $k(x, y)$ and to obtain $r$.

Estimating $k(x, y)$ by a physics-based approach is very hard, because measuring the actual values of $d(x, y)$ and $\theta(x, y)$ are very difficult. Instead, we utilize information provided in the image, where the appearance of a human face can be classified into normal and abnormal conditions. The normalcondition $(k(x, y)=1)$ is an actual appearance on parts of a human face in a normal lighting. The abnormal conditions $(k(x, y) \neq 1)$ is correspond to an appearance which is largely affected by the condition of direct lighting, i.e., cast shadow $(k(x, y)<1)$ and specular-reflection $(k(x, y)>1)$.

Equation (1) is valid only for certain conditions such as the presence of shadow $(k(x, y)<1)$ and non-shadow $(k(x, y)=1)$; the presence of specular reflection effect $(k(x, y)>1)$ is not taken into account. Although the shadow effect is more crucial problem than the specular effect, specular effects also potentially produce miss-recognition. Therefore, we consider all possible effects by dividing the range of human face intensity and assigning their properties as shown in Fig. 1. 


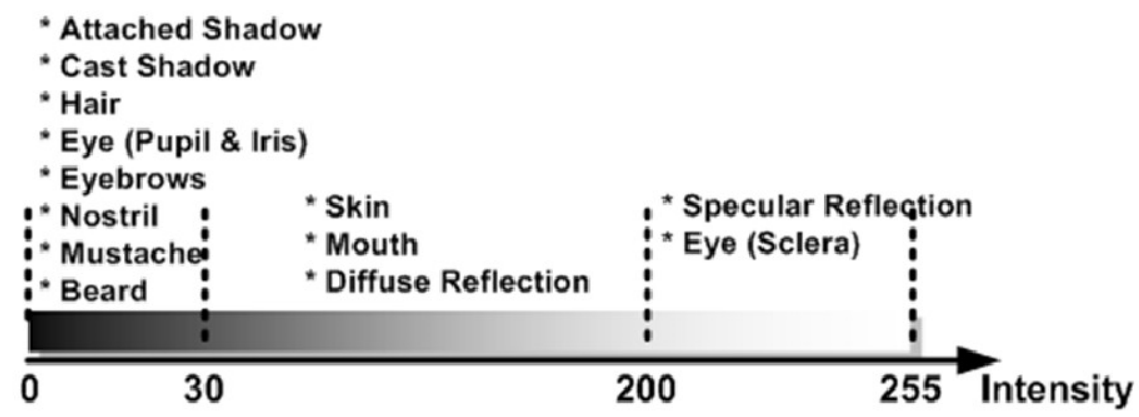

Figure 1. The possibility of human face intensity and its properties

Considering that the most part of human face is composed of skin, we segment human skin intensity based on [10-12] where human skin intensities are located between 30 and 200 (max. intensity 255). Using the maximum intensity of human skin, we make a rough approximation as follows:

$$
\hat{k}(x, y) \approx \frac{I(x, y)}{200}
$$

where $\hat{k}(x, y)$ is an approximated pixel's label at position $(x, y)$ that shows better segmentation of appearances on the human face. We then make a little modification to equation (1), that is,

$$
I(x, y)=\frac{(r+1)}{\hat{k}(x, y) r+1)} I^{*}(x, y),
$$

where we use a new pixel's label as $\hat{k}(x, y)$ instead of $k(x, y)$. The next step is to estimate the illumination ratior. Suppose we have three different appearances affected by the direct lighting as shown by Fig. 2. We can normalize shadow and specular reflection effects by knowing a native appearance and an actual appearance from a raw image. However, estimating

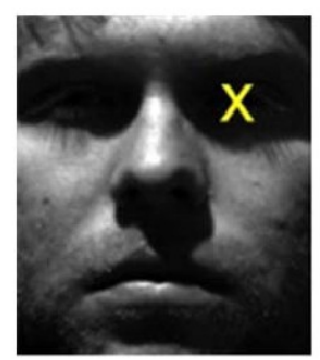

(a)

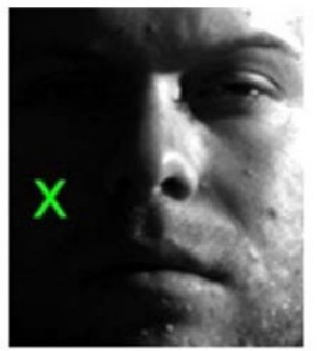

(b)

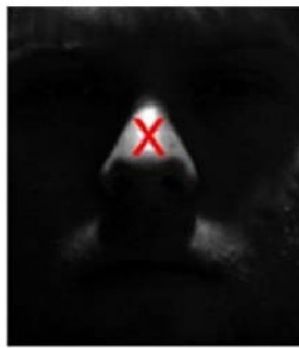

(c)

Figure 2. The differences of face's appearance due to direct lighting (Yale B face database) : (a) the pupil's appearance which is always dark even it is covered by shadow or not (yellow), (b) the cheek's appearance which is darker because of shadow effect (green), and (c) the nose's appearance which is brighter because of specular effect (red) 
a native face appearance from an illuminated image is very difficult. We can only estimate and model it by using some contrast enhancement to approximate the native face appearance. The actual face appearance from an image can be directly modeled from the image. Based on the approximated native face appearance and the actual face appearance information, we can determine $r$ for our illumination compensation model. For example, in the case of eye's pupil (Figure. 2(a)), the native appearance of the pupil is usually low. We can make a simple reasoning as follows: if the approximated native appearance is low and the actual appearance is low, then $r$ should be low. Another example, in the case of cheek (Figure. 2(b)), the native appearance of the cheek is usually medium. We can make a simple reasoning as follows: if the approximated native appearance is medium and the actual appearance is low, then $r$ should be high. Using such a kind of reasoning, we utilize optimized-Fuzzy to produce proper $r$ which is fed to our illumination compensation model for converting the raw image into a recognizable image.

\subsection{FACE IMAGE MODELLING}

\subsubsection{ACTUALFACE APPEARANCE MODELING}

To differentiate normal pixel, specular-reflected pixel and shadowed pixel, the actual appearance model is proposed. The direct illumination is highly dependent on the angle between the incoming light and the surface normal, which are hard to estimate from a single image. We therefore use the intensity value, that is,

$$
\operatorname{Act}(x, y)=I(x, y)
$$

where $I(x, y)$ is the pixel's intensity value at $(x, y)$ and $\operatorname{Act}(x, y)$ is its actual appearance. By using this value, we may divide the normal pixel, the shadowed pixel and the specular-reflected pixel, as shown in Fig. 3.

\subsubsection{APPROXIMATED NATIVEFACE APPEARANCE MODELING}

We apply a local contrast adjustment by using the BHE method to see if a pixel is inherently dark or is inherently bright but appears dark due to a

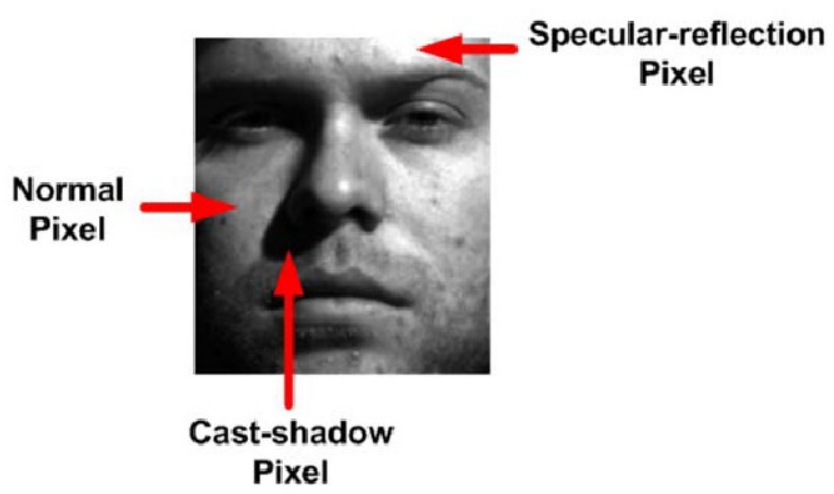

Figure 3. Normal, cast-shadow and specular-reflection detection (Yale B face database) 
shadow effect. A block size of $12 \times 12$ pixels is applied to the image and histogram equalization is performed. To avoid discontinuity between adjacent block, the block is moved in overlap to adjacent block every 6 pixel. Then, we apply weighted sum of neighboring adjacent blocks to smooth the boundaries in order to reduce blocking effect. Fig. 4 shows the illustration of BHE process. The BHE'd result is shown in Fig. 5.

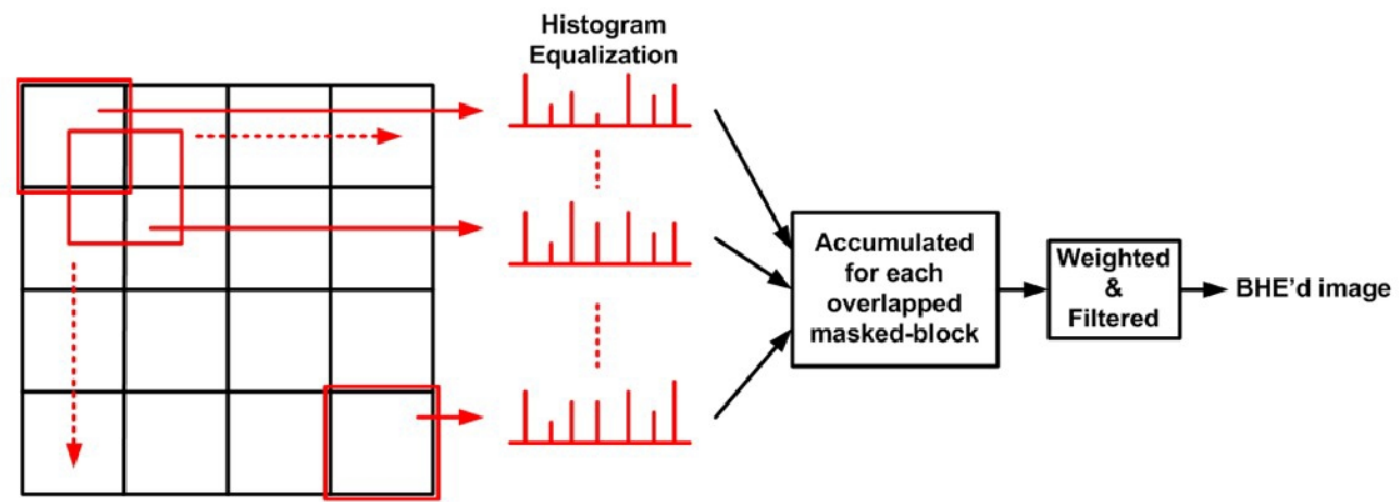

Figure 4. Illustration of Block-based Histogram Equalization
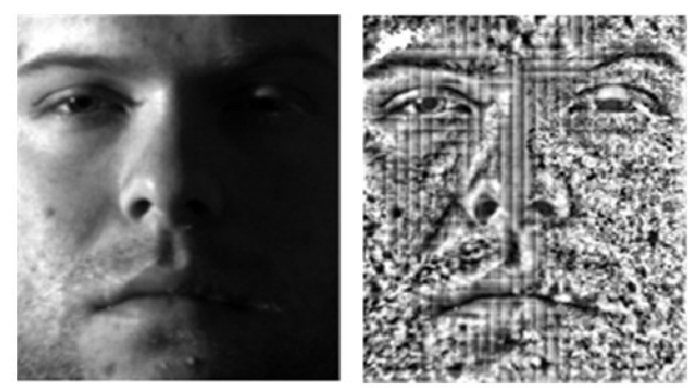

Figure 5. Block-based Histogram Equalization; Original image (Yale B face database, left), BHE'd image (right)

\section{FUZZY INFERENCE SYSTEM AND ITS OPTIMIZATION}

Our approach utilizes Fuzzy Inference System (FIS), which maps an input to an output using fuzzy logic approach. It is used to make reasoning of two models generated from an input image: approximated actual appearance model and current appearance model. FIS is chosen because it has capability to combine two informations which is supported by a prior knowledge called as a rule base. In general, FIS can be decomposed into three main steps: Fuzzyfication and membership function, inference engine and rule base, and defuzzyfication.

\subsection{FUZZYFICATION AND MEMBERSHIP FUNCTION}

The fuzzyfication converts a crisp input to a linguistic variable using the membership function. We use a triangular function because of two reasons: 
1. It takes a less computation time compared to others, and

2. According to [9], the illumination ratio $r$ can actually be obtained from linear equation.

Based on the triangular shape, we can derive a degree of membership function, that is,

$$
\mu_{m}=\left\{\begin{array}{rc}
\frac{x_{m}-a_{m}}{b_{m}-a_{m}} & \text { if } a_{m}<x_{m} \leq b_{m} \\
\frac{c_{m}-x_{m}}{c_{m}-b_{m}} & \text { if } b_{m}<x_{m} \leq c_{m} \\
0 & \text { if others }
\end{array}\right.
$$

where $\mu_{m}$ is a degree of m-th membership function, $x_{m}$ is the crisp input, $a_{m}$ is the left boundary of membership function, $b_{m}$ is the center of membership function and $c_{m}$ is the right boundary of membership function. The components of membership function of each model can be expressed as

$$
b_{m}=(m-1) \frac{255}{M-1}, a_{m}=b_{m-1}, c_{m}=b_{m+1}
$$

where $m=1,2, \ldots, M$ is the index of membership. Fig. 6 shows the shape of membership function.

\subsection{INFERENCE ENGINE AND RULE BASE}

We have two kinds of membership, $\mu_{A c t_{m}}$ and $\mu_{N a t_{m}}$ that represent degree of membership function of actual appearance model and approximated native appearance model, respectively. We used product of $\mu_{A c t_{m}}$ and $\mu_{N a t_{m}}$ instead of min-operator to form the rule as follows

$$
\boldsymbol{B}=\left[\mu_{\text {Act }} \mu_{\text {Nat }}\right]_{\{m=1,2, \ldots, M\}}
$$

where $\boldsymbol{B}=\left\{B_{1}, B_{2}, \ldots, B_{N}\right\}$ is a pair of inference of degree of membership function, with $N=M^{2}$. Since the number of inference is $N$, then the Fuzzy's rule $\boldsymbol{R}$ also has $N$ numbers of vector and can be described as follows

$$
\boldsymbol{R}=\left\{R_{1}, R_{2}, R_{3}, \ldots, R_{N}\right\}
$$

\subsection{DEFUZZYFICATION}

Finally, defuzzyfication is performed by converting the fuzzy output of the inference engine to crisp by adopting Center of Area (CoA) that is

$$
r=f(\boldsymbol{B}, \boldsymbol{R})=\frac{\sum_{n=1}^{N} B_{n} R_{n}}{\sum_{n=1}^{N} B_{n}}
$$

where $r$ is the illumination ratio and $n=1,2, \ldots, N$ is the number of inference $\boldsymbol{B}$ as well as the number of output of singleton function $\boldsymbol{R}$. 


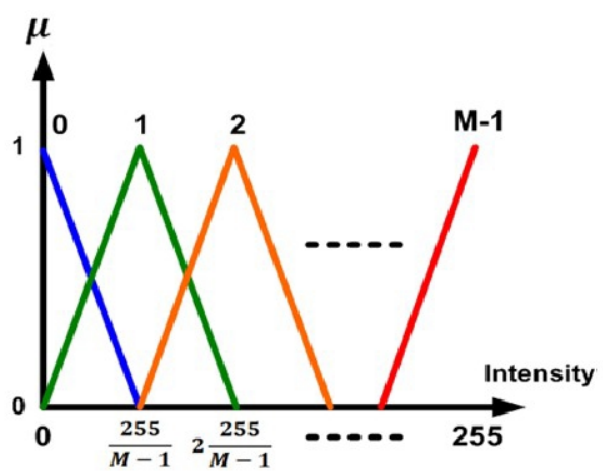

Figure 6. Fuzzy membership function

\subsection{OPTIMIZING RULE OF FUZZY}

The rule base is an important part of FIS to map the input into the output. Manual selection of rules is very difficult due to a very high and large variation of input, respect to the number of image's pixel and intensities. Optimizing the FIS rule base is therefore very important.

To produce a rule that satisfies all combinations as an optimal solution, GA is usedto find an optimal set of rule which fits to all illumination conditions. We utilize basic GA's components as follows

1. Initialize population

2. repeat

3. Evaluation

4. Reproduction

5. Crossover

6. Mutation

7. until : requirements are met

The initial population required at the beginning of the algorithm is a set of individual, $X$, that is consists of $N$-integer numbers of fuzzy's rule, $\boldsymbol{R}$, that is represents as gene generated by the random generator. Each set of individual is a representation of a solution to the optimization problem being addressed. Associated with each set of individual is a fitness value computed by the evaluation unit. This fitness value is a measure of the goodness of the solution that is represents.We use averaged-normalized cross correlation based distance to compute the fitness value, $f(X)$ as follows

$$
f(X)=\frac{1}{N_{\text {tim }}} \sum_{i=1}^{N_{\text {tim }}} \operatorname{corr}\left(I_{\text {norm }}^{1}, I_{\text {norm }}^{i}\right),
$$

where $f(X)$ is the fitness value for individual $X, N_{\text {tim }}$ is the number of training image, $I_{\text {norm }}^{1}$ is the $1^{\text {st }}$ normalized image, and $I_{\text {norm }}^{i}$ is the $i$-th normalized image, $i=1,2,3,4, \ldots, N_{t i m}$. The aim of the genetic operators is to transform this set of individual into sets with higher values. The reproduction 
operator performs a natural selection function to choose individuals with highest fitness value which has greater probability to be selected as a pair of parent to produce better offsprings. Then, crossover operator chooses pair of gene at a randomly selected point and to exchange their tails. The mutation operator randomly mutates the values of the same gene's number to optimize each individual. The process is iterated until required conditions are met (number of generation or determined fitness value). Fig. 7 shows our proposed system's block-diagram.

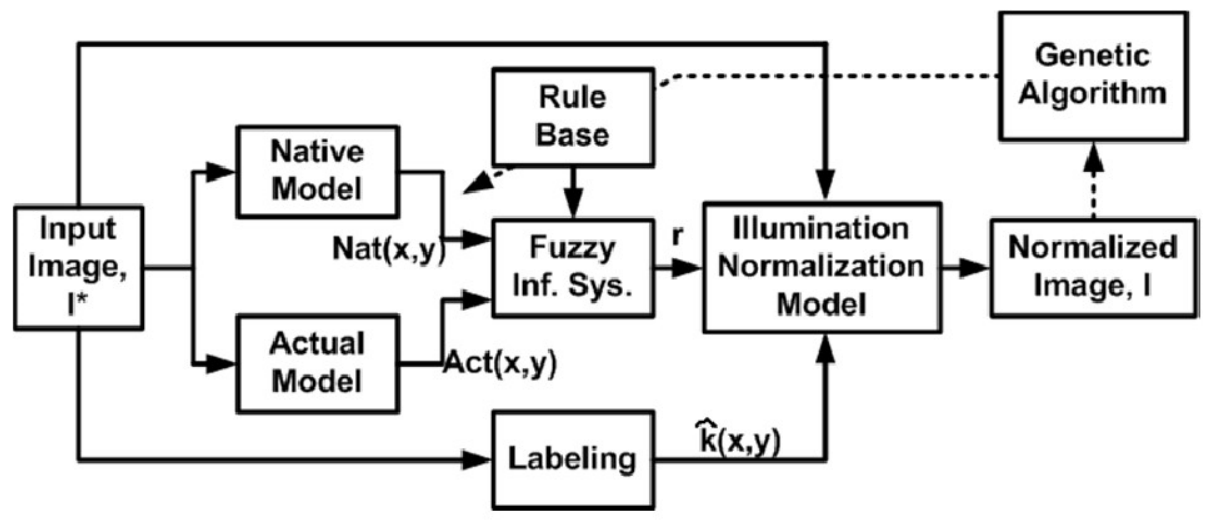

Figure 7. Proposed system

\section{EXPERIMENTS AND DISCUSSIONS}

We divide our experiments into two parts; (1) experiment with genetic algorithm to find the best rule and (2) evaluate and compareour method and other illumination compensation methods for still images in order to test the feasibility and performance using well known databases. We use Yale B Face database [13] and MIT Face database [14] as still image database to measure the recognition rate.

\subsection{EXPERIMENT WITH GENETIC ALGORITHM}

\subsubsection{EXPERIMENTAL SETUP}

For the optimization purpose, we choose a set of illuminations for one subject from Yale B face database [13]. This database is fairly representative with the availability of the most number of illumination effects. From the data set, we have 64 images with size of $192 \times 168$ pixels to be normalized. The main target is how to bring all different appearance images to be similar appearance images. To speed-up the computation time, we re-size the images into several scaled size and evaluated the performance of each scaled image's size for our illumination compensation method. The image size of $42 \times 48$ pixels is the smallest size for keeping the accuracy. So, we have $42 \times 48 \times 64=129,024$ variations of intensity. 


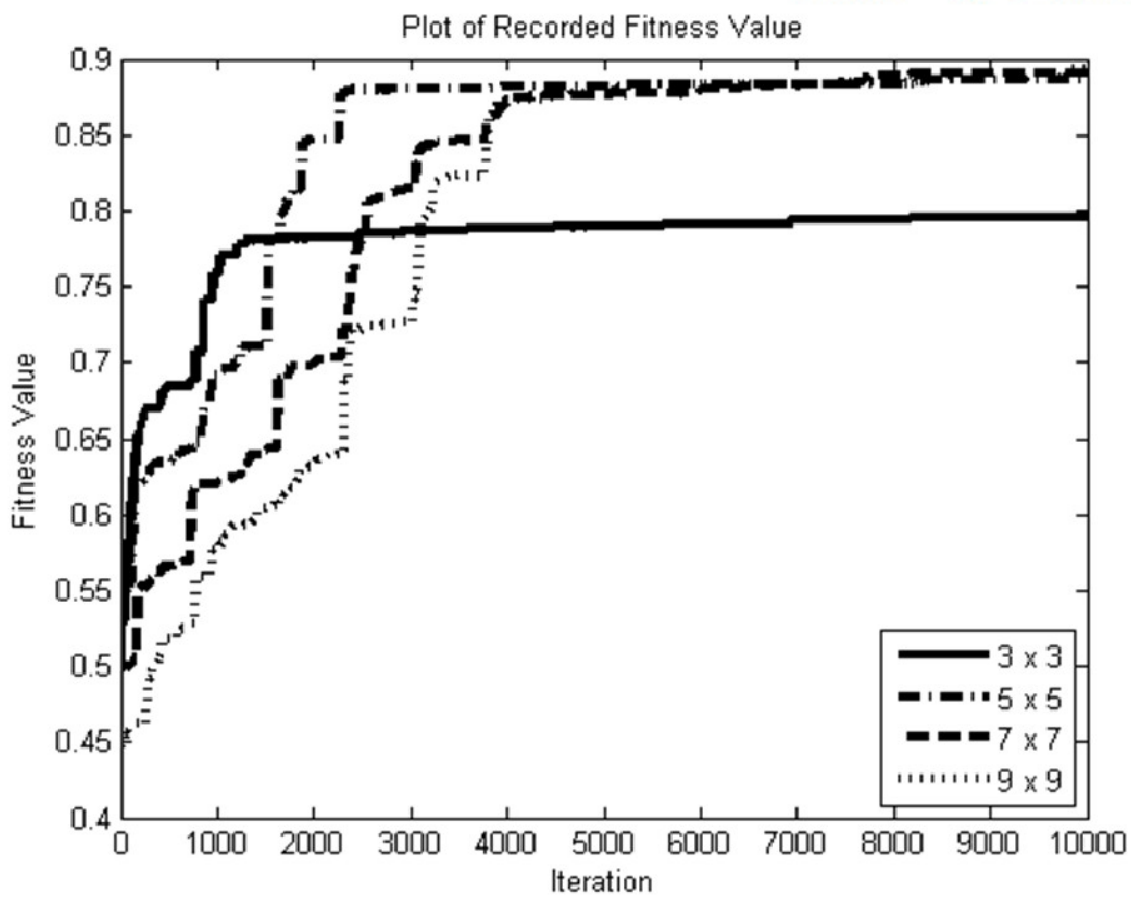

Figure 8. Evolution of GA's fitness value for different number of membership functions for 10,000 iterations

\subsubsection{EXPERIMENTAL RESULT}

We perform GA training for several different size of membership function, such as $3 \times 3,5 \times 5,7 \times 7$ and $9 \times 9$. Each process is iterated and recorded for 10,000 iterations as shown in Fig. 8. From the training, we got several rules that are shown by Figure 9. Respect to the evolution of GA's fitness values as shown in Figure8, the training result of $3 \times 3$ fuzzy membership function achieves the lowest final fitness value. This means that the optimization result is still not good. The training result of $7 \times 7$ and $9 \times 9$ of fuzzy membership functions achieve good final fitness value as well as $5 \times 5$, but they experience slower transition. This means that they may experience local optimum problem in specific iteration step. Otherwise, $5 \times 5$ fuzzy memberships function achieves better result as indicated by the rapid transition and high fitness value.

Table 1. Recognition rate of Yale B face database (\%)

\begin{tabular}{crrr}
\hline No & Method & NN & Eigenfaces \\
\hline 1 & Fuzzy 3x3 & 97.66 & 84.38 \\
2 & Fuzzy 5x5 & $\mathbf{9 9 . 8 4}$ & $\mathbf{9 8 . 7 5}$ \\
3 & Fuzzy 7x7 & 93.28 & 74.69 \\
4 & Fuzzy 9x9 & 99.69 & 89.06 \\
\hline
\end{tabular}

Our hypothesis is proven after carrying out experiments, where we get poor results when we test $3 \times 3,7 \times 7$ and $9 \times 9$ membership functions using eigenfaces (see Table 1). $3 \times 3$ memberships function has too little variation of rule that makes it too stuffy, produce poor and rough normalization result 
and still leaving traces of the shadow. Otherwise, $7 \times 7$ and $9 \times 9$ membership functions have too many variation of rule that makes the normalization result too "flat" by reducing actual boundary among face parts (such as eye, mouth, etc) and take longer computation time. Finally, we decided to choose $5 \times 5$ memberships function as our best solution. The comparison results can be seen in Figure10.

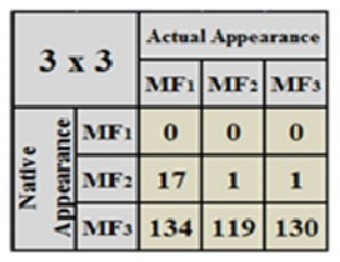

(a)

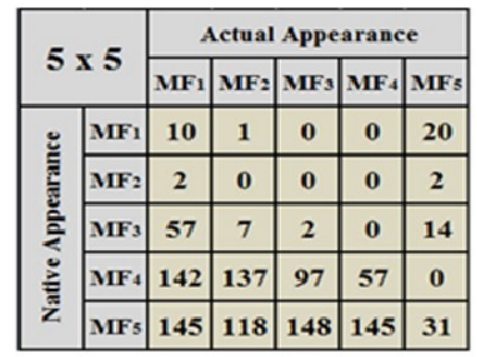

(b)

\begin{tabular}{|c|c|c|c|c|c|c|c|c|}
\hline \multirow{2}{*}{\multicolumn{2}{|c|}{$7 x$}} & \multicolumn{7}{|c|}{ Ietual Appearance } \\
\hline & & MF1 & $\mathrm{MF}=$ & $\mathrm{MF} 3$ & MF 4 & MIFs & MF6 & \\
\hline \multirow{7}{*}{ 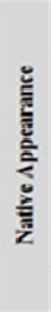 } & & 112 & 56 & 31 & 16 & 1 & 0 & 10 \\
\hline & MF $=$ & 63 & 5 & & $\mathbf{0}$ & $\mathbf{0}$ & 0 & 14 \\
\hline & MFs & 120 & 107 & 70 & 25 & 8 & 0 & 11 \\
\hline & $\mathrm{MF}_{4}$ & 123 & 111 & 106 & 118 & 87 & $\mathbf{0}$ & 13 \\
\hline & MFs & 142 & 145 & 125 & 105 & 107 & 0 & 13 \\
\hline & MFo & & $I^{2}$ & 144 & 117 & 129 & $\mathbf{0}$ & 77 \\
\hline & SEF- & 107 & 1 & 111 & 120 & 114 & 1 & 76 \\
\hline
\end{tabular}

(c)

\begin{tabular}{|c|c|c|c|c|c|c|c|c|c|c|}
\hline \multirow{2}{*}{\multicolumn{2}{|c|}{$9 \times 9$}} & \multicolumn{9}{|c|}{ Actual Appearance } \\
\hline & & \multirow{2}{*}{\begin{tabular}{|c|} 
MF 1 \\
96 \\
\end{tabular}} & \multirow{2}{*}{\begin{tabular}{|c|} 
MF: \\
55 \\
\end{tabular}} & \multirow{2}{*}{\begin{tabular}{|c|} 
MFs \\
9
\end{tabular}} & \multirow{2}{*}{\begin{tabular}{|c|} 
MF 4 \\
8
\end{tabular}} & \multirow{2}{*}{\begin{tabular}{|c|} 
MFs \\
1 \\
\end{tabular}} & \multirow{2}{*}{\begin{tabular}{c|}
$\mathrm{MF}_{6}$ \\
1
\end{tabular}} & \multirow{2}{*}{\begin{tabular}{|c|}
$\mathrm{MF}$, \\
1 \\
\end{tabular}} & \multirow{2}{*}{\begin{tabular}{|c|}
$\mathrm{MFs}$ \\
0
\end{tabular}} & \multirow{2}{*}{\begin{tabular}{|l} 
MFs \\
124
\end{tabular}} \\
\hline \multirow{9}{*}{ 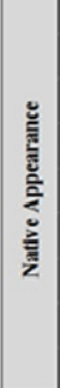 } & MF1 & & & & & & & & & \\
\hline & MF $=$ & 18 & 0 & 2 & 0 & o & $\mathbf{0}$ & 0 & 0 & 137 \\
\hline & MFs & 108 & 48 & 19 & 11 & 7 & 2 & 2 & 2 & 136 \\
\hline & $\mathrm{MF}_{4}$ & 124 & 117 & 120 & 92 & 59 & 49 & 6 & 81 & 89 \\
\hline & MFs & 104 & 122 & 80 & 89 & 141 & 115 & 83 & 86 & 138 \\
\hline & MF。 & 105 & 141 & 137 & 94 & 102 & 111 & 106 & 132 & 129 \\
\hline & MF, & 137 & 123 & 103 & 114 & 134 & 87 & 123 & 64 & 127 \\
\hline & MFs & 122 & 111 & 134 & 114 & 107 & 129 & 113 & 55 & 92 \\
\hline & MF, & 148 & 79 & 127 & 81 & 114 & 127 & 124 & 7 & 73 \\
\hline
\end{tabular}

(d)

Figure 9. Fuzzy rules that heuristically generated by GA; (a) $3 \times 3$ membership function, (b) $5 \times 5$ membership function, (c) 7x7 membership function and (d) $9 \times 9$ membership function

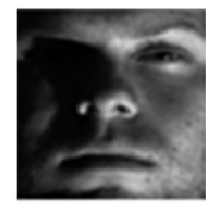

(a)

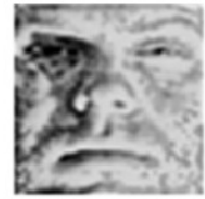

(b)

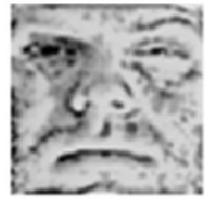

(c)

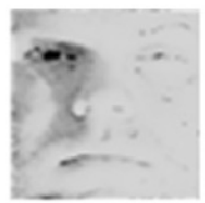

(d)

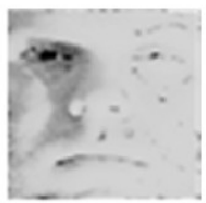

(e)

Figure 10. Comparison results of different size of membership function; (a) original image, (b) $3 \times 3$, (c) $5 \times 5$, (d) $7 \times 7$ and (e) $9 \times 9$

\subsection{EVALUATING METHODS IN COMPARISON}

\subsubsection{EXPERIMENTAL SETUP}

Yale B face database contains 38 subjects with 64 illumination conditions under single illumination, while MIT face database contains 10 
subjects with 36 illumination conditions with several numbers of illuminations. Only the frontal face images with illumination variation are selected and manually cropped. Each image is cropped in size of $168 \times 192$ pixels. To simplify our analysis process, we divided Yale B face database into five subsets. Each subset is formed based on its illumination angles.The first subset represents images with azimuth or elevation angle $\phi<12^{\circ}$, while the second subset represents images with angle $15^{\circ} \leq \phi \leq 30^{\circ}$, the third subset represents images with angle $35^{\circ} \leq \phi \leq 50^{\circ}$, the fourthsubset represents images with angle $60^{\circ} \leq \phi \leq 77^{\circ}$, and the fifth subset represents images with angle $\phi>77^{\circ}$. This fraction is shown in Fig. 11. We employed two kinds of classifier, the Nearest Neighbor (NN) with correlation coefficient as its distance measurement and Eigenfaces [15] with 10 principal components.

Subset 1

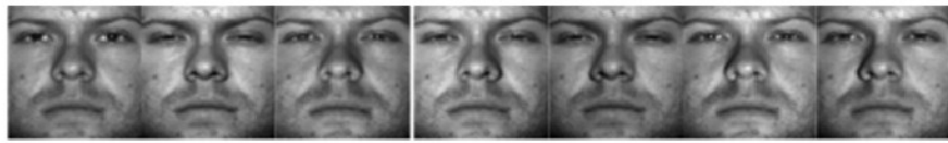

Subset 2

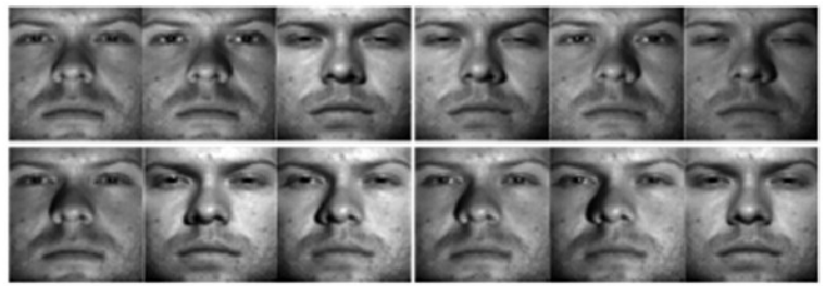

Subset 3

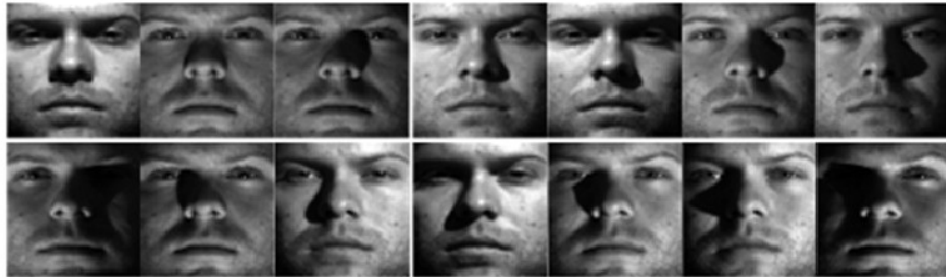

Subset 4
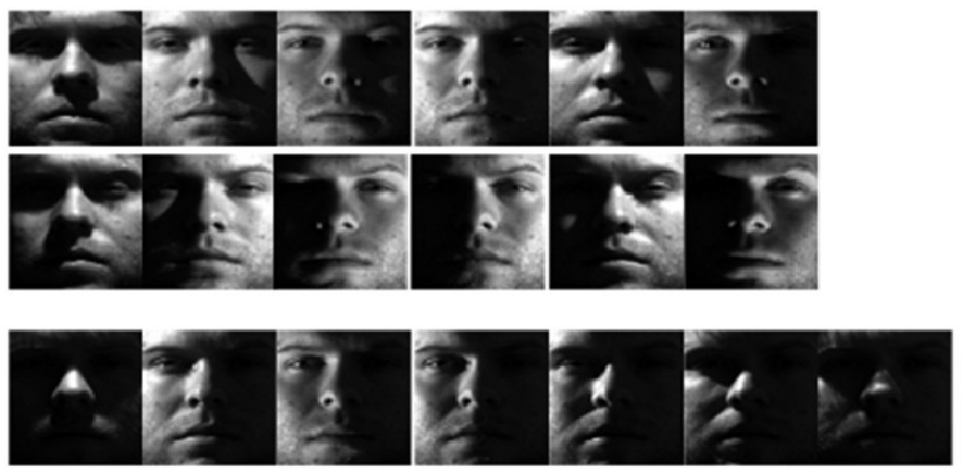

Subset 5
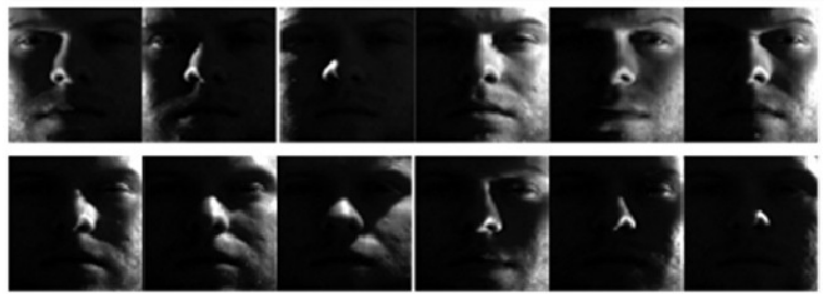

Figure 11. The fraction of Yale B face database subset based on its illumination angle 
In order to test the performance, we use only one frontally illuminated image as a template from 10 persons in Yale B face database and 10 persons in MIT face database. All the images have been converted to normalized images by using the proposed method before the recognition process. Each normalized image is resized to $64 \times 64$ pixels. As comparison methods, FuzzyRetinex (FR), Adaptive Smoothing Retinex (ASR), and Optimized-SQI (OptSQI) are included. Since each method contain some parameters to be set, we summarize the parameter setting as shown in table 2 .

Table2. Parameter setting of proposed method and other methods for comparison

\begin{tabular}{llr}
\hline \multicolumn{1}{c}{ Method } & \multicolumn{1}{c}{ Parameters } & Value \\
\hline FR & Filter size (Gaussian) & 3 \\
& Fuzzy membership function & Triangular, 5x5 \\
ASR & Smoothing iteration (T) & 50 \\
Opt-SQI & Filter size (Gaussian) & $3,5,7,9,11,13,15,17$ \\
& $\beta_{k}$ & $1,1 / 15,2 / 15,7 / 15,1,1,1,3 / 15$ \\
& $m_{k}$ & $4 / 15,1 / 15,1 / 3,0,4 / 15,14 / 15,14 / 15$, \\
& $\alpha$ & $3 / 5$ \\
Ours & Block size (Native & 0.98 \\
& Appearance) & 12 \\
& Fuzzy membership function & Triangular, 5x5 \\
& Fuzzy rule & See Figure 9 \\
\hline
\end{tabular}

\subsubsection{EXPERIMENTAL RESULT}

In this section, we re-implement the other illumination compensation methods and compare them with our method. Figure 12 shows the illumination compensation results for Yale B face database of all methods including ours. We evaluate the results using $\mathrm{NN}$ and Eigenfaces with 10 principal components. Recognition result of each method is shown in table 3 and table 4 for $\mathrm{NN}$ and Eigenfaces, respectively.

FR achieves worst results since it can not control its tonal rendition for both normal and harsh illuminated faces. The final appearances fail to be recognized using NN as well as Eigenfaces. ASR and Opt-SQI achieve similar results, where they can perform well for all subsets. Our approach is successfully removes the shadow and the specular-reflection effects in the converted face image by controlling final skin intensities into similar value and retain the other intensity that should not be raised, e.g., eyes, eyebrows, line of mouth, etc. Our method also produces very strong texture so it may be useful for classification. We have proved that our approach is able to achieve better performance for both $\mathrm{N}$ and Eigenfaces. 
Table3. Recognition rate of Yale B face database using Nearest Neighbor (\%)

\begin{tabular}{llrrcccc}
\hline No & Method & Subset & Subset & Subset & Subset & Subset & Average \\
& & $\mathbf{1}$ & $\mathbf{2}$ & $\mathbf{3}$ & $\mathbf{4}$ & $\mathbf{5}$ & \\
\hline 1 & FR & 84.29 & 84.17 & 81.43 & 85.00 & 85.26 & 84.03 \\
2 & ASR & $\mathbf{1 0 0 . 0 0}$ & $\mathbf{1 0 0 . 0 0}$ & 95.71 & 95.83 & 96.32 & 97.57 \\
3 & Opt-SQI & $\mathbf{1 0 0 . 0 0}$ & $\mathbf{1 0 0 . 0 0}$ & 97.14 & 95.83 & 95.26 & 97.65 \\
4 & Ours & $\mathbf{1 0 0 . 0 0}$ & $\mathbf{1 0 0 . 0 0}$ & $\mathbf{9 9 . 2 9}$ & $\mathbf{9 8 . 3 3}$ & $\mathbf{9 9 . 4 7}$ & $\mathbf{9 9 . 4 2}$ \\
\hline
\end{tabular}

Table4. Recognition rate of Yale B face database using Eigenfaces (\%)

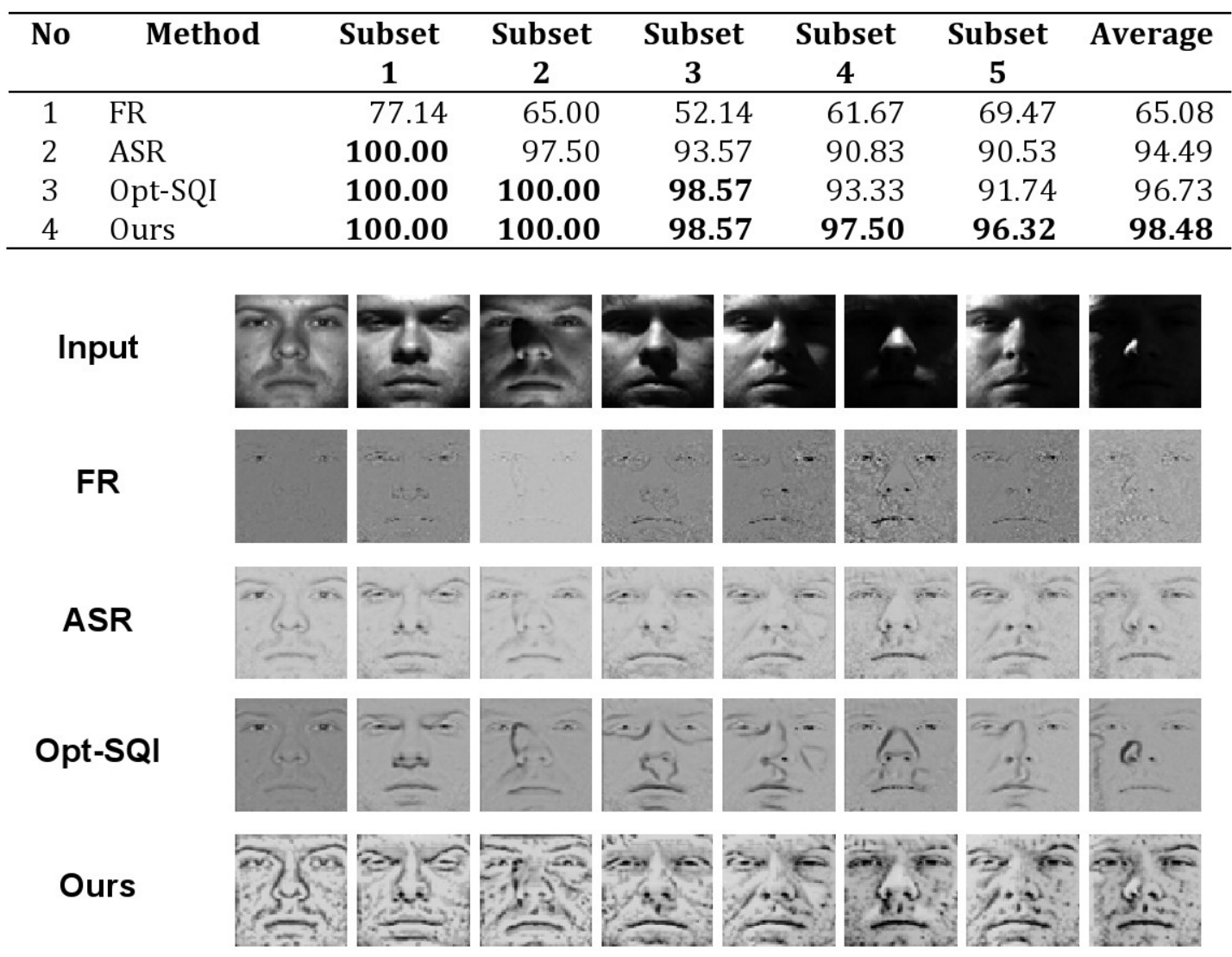

Figure 12. Comparison of illumination compensation methods for Yale B face database

The next experiment is using MIT face database that contains several light illuminated images. The lighting effects are not as difficult as Yale B face database. As our prediction, almost all illumination compensation methods successfully achieve perfect performance (see Table 5 and Figure 13). The result shows that each compensation method is feasible for moderate illumination condition. 
Table 5. Recognition rate of MIT face database (\%)

\begin{tabular}{clcr}
\hline \multirow{2}{*}{ No } & \multirow{2}{*}{ Method } & \multicolumn{2}{c}{ Classifier } \\
\cline { 3 - 4 } & & NN & Eigenfaces \\
\hline 1 & FR & 100.00 & 100.00 \\
2 & ASR & 100.00 & 100.00 \\
3 & Opt-SQI & 100.00 & 100.00 \\
4 & Ours & 100.00 & 100.00 \\
\hline
\end{tabular}

Input

FR
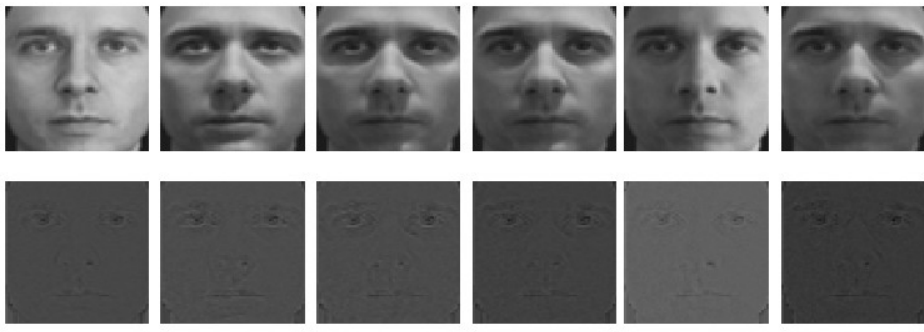

ASR
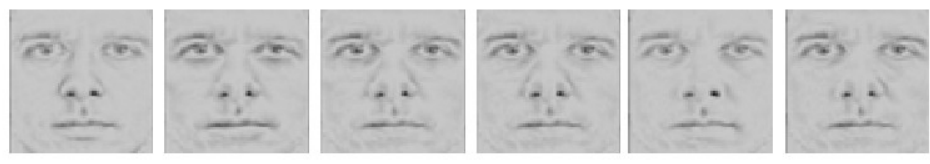

Opt-SQI
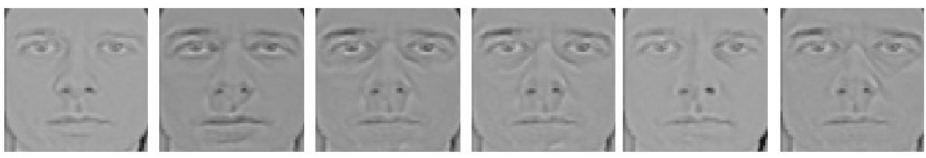

Ours
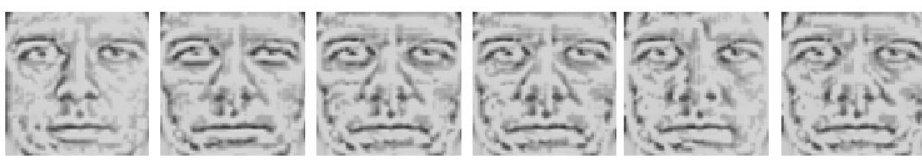

Figure 13. Comparison of illumination compensation methods for MIT face database

\subsubsection{COMPUTATION TIME}

We do not just concentrate on the performance of the method, but also on the speed of computation.We evaluate the speed of computation of all methods that is obtained by averaging the computation time of 640 images in Yale B face database and 360 images in MIT face database. Evaluation is conducted using Microsoft Visual C++ runs on a personal computer system equipped with $2.66 \mathrm{GHz}$ Intel processor supported by $2 \mathrm{~GB}$ of RAM. We got the fact that our proposed system requires only $13.4 \mathrm{~ms}$ to complete its calculation, while FR gets $8.93 \mathrm{~ms}$ and ASR obtains $73.36 \mathrm{~ms}$. Opt-SQI notes the longest computation time, $256.7 \mathrm{~ms}$, to convert one image.

\section{CONCLUSION AND FUTURE WORK}

In this paper, we have showed superior results of the optimized Fuzzy rules for Fuzzy-based illumination compensation problem in face recognition. The proposed method seems very promising since it only requires very low computation time with high performance. This method is able to produce better converted image where details of face can still be seen 
clearly. Face's textures are strengthened and very helpful for face detector to distinguish between human face and others.

For the next plan, we are going to increase our system capability in order to detect and recognize more than one person in a single frame, so it can accommodates real situation where many person are exist surrounding the robot.

\section{ACKNOWLEDGEMENTS}

We would like to thank to Directorate General of Higher Education (DIKTI)for the contribution tosupportscholarships.

\section{REFERENCES}

[1] L.A. Zadeh, Outline of a New Approach to The Analysis of Complex Systems and Decision Processes, IEEE transactions Systems, Man, Cybernetics, SMC-3, pp. 28-44, 1973.

[2] J.H. Holland, Genetic Algorithms, Scientific American, pp.66-72, 1992.

[3] B.S.B Dewantara, J. Miura, Fuzzy-based Illumination Normalization for Face Recognition, IEEE Workshop on ARSO, pp. 131-136, 2013.

[4] G.P. Nam, K.R. Park, New Fuzzy-based Retinex Method for the Illumination Compensation of Face Recognition, Int. Journal of Advanced Robotic System. Vol. 9, pp.103, 2012.

[5] Y.K. Park, S.L. Park, J.K. Kim, Retinex Method Based on Adaptive Smoothing for Illumination Invariant Face Recognition, Signal Processing, Vol. 88, pp.1929-1945, 2008.

[6] C.A. Perez, L.E. Castillo, Illumination Compensation for Face Recognition based on Genetic Optimization of the Self-Quotient Image Method, International Symposium on Optomechatronic Technologies, pp.322-327, 2009.

[7] K.R. Singh, M.A. Zaveri, M.M. Raghuwanshi, Rough Membership Function Based Illumination Classifier for Illumination Invariant Face Recognition, Applied Soft Computing, Vol. 12, pp.4105-4117, 2013.

[8] E.H. Mamdani, Application Of Fuzzy Logic To Approximate Reasoning Using Linguistic Synthesis, IEEE Transactions on Computer C, Vol. 26, No. 12, pp.1182-1191, 1977.

[9] R. Guo, Q. Dai, D. Hoeim, Single-Image Shadow Detection and Removal using Paired Regions, IEEE Conference on Computer Vision and Pattern Recognition, pp.2033-2040, 2011.

[10] P. Peer, F. Solina, An Automatic Human Face Detection Method, Proceedings of the 4th Computer Vision Winter Workshop. Pp.122-130, 1999.

[11] A. Elgammal, C. Muang, D. Hu, Skin Detection - A Short Tutorial, Encyclopedia of Biometrics by Springer-Verlag Berlin Heidelberg, 2009. 
[12] G. Grove, C. Zerweck, J. Damia, Human Skin Coloration using the RGB Color Space Model, The 4th International Symposium of L'Oreal Institute for Ethnic Hair and Skin Research, 2007.

[13] A.S. Georghiades, P.N. Belhumeur, D.J. Kriegman, From Few to Many: Illumination Cone Models for Face Recognition under Variable Lighting and Pose, IEEE Trans. Pattern Analysis and Machine Intelligence. Vol. 23, No. 6, pp.643-660, 2001.

[14] B. Weyrauch, J. Huang, B. Heisele, V. Blanz, Component-based Face Recognition with 3D Morphable Models, Int. Conf. on CVPR Workshop, pp.85, 2004.

[15] M.A. Turk, A.P. Pentland, Eigenfaces for Recognition, Journal of Cognitive and Neuroscience, Vol. 3, pp.71-86, 1991. 